\title{
Arzanà
}

Cahiers de littérature médiévale italienne

$19 \mid 2017$

Varia

\section{Il sodalizio franco-italiano di André Pézard e Gianfranco Contini : l'ermeneutica filologica come traduzione}

Le partenariat franco-italien d'André Pézard et Gianfranco Contini :

l'herméneutique philologique comme traduction

\section{Viviana Agostini-Ouafi}

\section{OpenEdition}

\section{Journals}

Edizione digitale

URL: http://journals.openedition.org/arzana/1068

DOI: $10.4000 /$ arzana.1068

ISSN: 2429-9499

\section{Editore}

Presses Sorbonne Nouvelle

\section{Edizione cartacea}

Paginazione: 96-103

ISSN: $1243-3616$

Questo documento vi è offerto da Université de Caen Normandie

$$
\mathrm{U} \sim \stackrel{\mathrm{N}}{\mathrm{N}} \mathrm{N}
$$

Notizia bibliografica digitale

Viviana Agostini-Ouafi, « II sodalizio franco-italiano di André Pézard e Gianfranco Contini :

l'ermeneutica filologica come traduzione », Arzanà [Online], 19 | 2017, online dal 23 octobre 2017, consultato il 04 juin 2019. URL : http://journals.openedition.org/arzana/1068 ; DOI : 10.4000/ arzana.1068 


\title{
Il sodalizio franco-italiano di André Pézard e Gianfranco Contini : l'ermeneutica filologica come traduzione
}

\author{
Le partenariat franco-italien d'André Pézard et Gianfranco Contini : \\ l'herméneutique philologique comme traduction
}

I legami stabiliti intorno all'opera di Dante dai critici e filologi romanzi A. Pézard e G. Contini costituiscono un aspetto trascurato delle relazioni franco-italiane del Novecento. Il primo ha operato alla fine degli anni 1970 affinché il collega fosse eletto come membro associato straniero all'Institut de France, ma la sua scoperta della critica continiana risale al 1946. Quando esce il lavoro curato da Contini Poeti del Duecento, Pézard è impegnato nella sua traduzione completa dell'opera di Dante. La recensione a questo lavoro da lui redatta nel 1962 aiuta a capire quale idea della filologia moderna, strutturalista e ermeneutica, li animi. Lo studio di Contini Filologia ed esegesi dantesca e l'Avertissement pezardiano della Pléiade, pubblicati nel 1965, completano questo quadro in cui interpretazione filologica e traduzione assumono, in nome di Dante, il loro senso profondo.

Parole chiave : filologia, ermeneutica, traduzione, Dante, Pézard, Contini

Les liens tissés autour de l'œuvre de Dante par les critiques et philologues romanistes A. Pézard et G. Contini constituent un aspect négligé des relations franco-italiennes du $\mathrm{XX}^{\mathrm{e}}$ siècle. Le premier a œuvré dans les années 1970 pour que son confrère soit élu comme Membre Associé Étranger à l'Institut de France, mais sa découverte de la critique continienne remonte à 1946. Lorsque paraît l'ouvrage édité par Contini Poeti del Duecento, Pézard est occupé à sa traduction complète de l'œuvre de Dante. Le compte rendu de cet ouvrage qu'il rédige en 1962 aide à comprendre quelle idée de la philologie moderne, structuraliste et herméneutique, les anime. L'étude de Contini Filologia ed esegesi dantesca et l'Avertissement pézardien de la Pléiade, parus en 1965, complètent ce tableau où interprétation philologique et traduction prennent, au nom de Dante, tout leur sens.

Mots clés : philologie, herméneutique, traduction, Dante, Pézard, Contini

ianfranco Contini (1912-1990) è uno dei più grandi critici dell'Italia del Novecento, ma Il recente numero X di « Ermeneutica letteraria » mette proficuamente in evidenza anche l'apporto della cultura francese nella sua formazione e nella sua opera di filologo e critico militante'.

1. Paolo LEONCINI (dir.), "Gianfranco Contini entre France et Italie : philologie et critique », Ermeneutica letteraria, X (2014). V. la nota critica da noi dedicata a questo numero in Viviana AGOSTINI-OUAFI, Antonio 
Siamo convinti del resto che valorizzare la Francia di Contini può permetterci di capire meglio i molteplici contatti che nel secolo scorso hanno caratterizzato le relazioni tra i due paesi ${ }^{2}$. Nel corso dei nostri studi, incentrati in particolare sul proustismo italiano critico e traduttivo, abbiamo ineluttabilmente incontrato varie volte Contini ${ }^{3}$, tuttavia solo in questi ultimi anni, lavorando sui testi editi o archivistici di un dantista e traduttore francese come André Pézard (1893-1984), abbiamo sentito l'esigenza di approfondire l'insegnamento di questo maestro. In effetti non è la critica degli Ossi di seppia di Montale o delle intermittences du cœur di Proust, aspetti magistralmente indagati da Giacomo Debenedetti quando Contini era ancora adolescente ${ }^{4}$, che ci sembra costituire l'apporto principale del suo pensiero critico. La questione filologica degli scartafacci, da lui sollevata fin dal 1937 per l'Ariosto, poi subito indagata in modo ineccepibile per Dante e Petrarca, e trasferita poi senza tema anche nello studio di autori moderni e contemporanei, compreso Proust, ci sembra invece frutto della sapiente genialità di un filologo che è stato al contempo, per dirla con Pier Vincenzo Mengaldo, uno "strutturalista precoce o avanti lettera "s. Date tali premesse, vorremmo qui tentare di gettare un po' di luce su un capitolo oggi abbastanza trascurato delle relazioni franco-italiane del Novecento, capitolo le cui problematiche sono sintetizzabili nella seguente domanda : nella Francia di Contini, che posto ha occupato Pézard? Questione, come vedremo, anche del tutto reversibile : nella Francia di Pézard, che posto ha occupato Contini? Cercheremo dunque di capire quali relazioni abbiano intessuto questi due grandi filologi che si sono imposti, ciascuno nel suo paese e quasi negli stessi anni, come emeriti dantisti. Chissà se non scopriremo - tolta la polvere del tempo, del rimosso e dell'oblio - che il maestro Contini aveva anche colti e appassionati seguaci in Francia e che i ponti franco-italiani erano forse molto più solidi, frequentati e costruttivi di quanto oggi si voglia o si possa immaginare.

Un binomio concettuale, esplicitato da Contini nel suo scritto del 1965 Filologia ed esegesi dantesca, ci sembra riassumere la sua concezione organica della critica letteraria : fruizione dell'opera, lettura, espressione pura da una parte, e giudizio, commento, rapporti linguistici dall'altra possono essere analogicamente rappresentati dal binomio anglofono "Growth AND Structure ", rispetto al quale lo sforzo della linguistica moderna non sarà semplicemente di alternare, bensì di colle-

LAVIERI (dir.), « Poétiques des archives. Genèse des traductions et communautés de pratique », Transalpina, 18 (2015), p. 201-204.

2. Ricco di possibili sviluppi è in questo senso lo studio di Carlo Ettore COLOMBO, «Les débuts italo-provençaux de Gianfranco Contini ", Ermeneutica letteraria, cit., p. 33-39.

3. Per esempio, per la relazione problematica, soprattutto negli anni 1920-1950, dei traduttori e dei proustiani italiani con Croce (v. Viviana AgOstini-OuAfi, Poetiche della traduzione. Proust e Debenedetti, Modena, Mucchi, 2010, p. 41-46), oppure per la questione filologico-editoriale degli interventi non autorizzati di Jacques Rivière sui testi di Proust (v. ID., "Il proustimo di Corrado Alvaro ", Fronesis, 8 [2008], p. 90-91).

4. Le prime poesie di Montale escono nel 1922 su una rivista, "Primo tempo ", la cui sede è nell'abitazione stessa di Debenedetti, e il denso meta-racconto riflessivo pubblicato dal critico nel 1926, Riviera amici (in Amedeo e altri racconti, Torino, Edizioni del Baretti) è fin dal titolo un omaggio esplicito ad Ossi di seppia, raccolta poetica di cui aveva poco prima riletto personalmente le bozze! Quanto alle intermittences, l'eccellente analisi che Contini propone nel 1953 concerne volutamente il solo Jean Santeuil, che è in effetti l'infanzia della Recherche, ma non la Recherche. Per ripercorrere la genesi delle intermittences e coglierne tutto il senso, sono utili soprattutto i vari abbozzi di prefazione scritti verso il 1908 da Proust per il Contre Sainte-Beuve (già luminosa in tal senso è l'edizione difettosa di Bernard De Fallois, Parigi, Gallimard, 1954, p. 60-61).

5. Pier Vincenzo MENGaldo, Profili di critici del Novecento, Torino, Bollati Boringhieri, 1998, p. 53. V. il preciso ritratto di Contini, filologo strutturalista e precursore della genetica testuale, che Claude Perrus ricostruisce in ineccepibile prospettiva storica, esemplificandone i principi e la metodologia critica proprio sulle paperoles di Proust : "Gianfranco Contini et l'approche de l'œuvre 'in fieri' ", in Dominique BUDOR, Claude PERRUS (dir.), Le texte : genèse, variantes, édition, Arzanà, 5 (2000), p. 15-16.

6. Gianfranco CONTINI, Varianti e altra linguistica. Una raccolta di saggi (1938-1968) [1970], Torino, Einaudi, 1979 , p. 410. Tale binomio appare forse per la prima volta nel titolo di un libro del linguista danese Otto Jespersen pubblicato nel 1905. 
gare il piano dello Sviluppo (linguistica diacronica) con quello della Struttura (linguistica sincronica). Per Contini anche la più moderna critica dantesca realizza « un avvicinamento della linea espressiva e, per così dire, esecutiva e della linea esegetica e sistematica, interrompendo la prima per stabilire connessioni del secondo ordine e ritornando alla lettura diretta con questo sottofondo di esperienze $»^{7}$. Lo sviluppo espressivo, della scrittura o della lettura, è dinamico, iscritto nella storia, invece la struttura è un insieme di corrispondenze sistematiche, in apparenza statiche e sincroniche, che sono strettamente connesse al commento esegetico : ognuno dei due piani aiuta a capire l'altro, in un rapporto di fatto dialettico. L'esegesi strutturalista è quindi in costante interconnessione con lo sviluppo espressivo filologico. Egli propone poi una serie di operazioni che dovrebbe prefiggersi questa critica dantesca moderna, definibile secondo lui non tanto come una critica " stilistica " o «strutturale ", ma come una « critica verbale».

Constatiamo così che con la prima operazione - « verifiche puntuali dei canoni retorici, per inserzione nella continuità classica " - Contini introduce nel suo approccio critico diacronico dell'espressività anche la storia della letteratura, ovvero la tradizione letteraria col suo vasto e polimorfo dialogo intertestuale : il piano sistemico invade subito allora quello espressivo. Gli stilemi generici o autoriali, oggetto sovente di prestito intertestuale, a quale piano appartengono? Un poliglotta dello stampo di Contini naviga poi a proprio agio tra lingue morte e vive, tra idiomi subalterni e egemoni ${ }^{8}$. Per questo motivo, nella quarta operazione da lui proposta - " nuove ed esasperate auscultazioni della lettera finché essa non liberi una traduzione inedita " - convoca niente di meno che la traduzione. Si tratta di un'idea di " traduzione " tipica del pensiero filosofico ermeneutico di matrice idealistica, in cui anche sul piano endolinguistico (seppure dall'italiano antico a quello moderno) interpretare significa tradurre'. Il mutamento semantico, d'altronde, come dimostra con la sua analisi del sonetto Tanto gentile e tanto onesta pare ${ }^{10}$, può intaccare il senso della parola senza modificarne in nulla la forma, l'opera implacabile del tempo basta e avanza, esattamente come prima di lui ci ha insegnato anche Jorge Louis Borges ${ }^{11}$. Lo sviluppo espressivo, della scrittura o della lettura, è sempre quindi per lui una forma dinamica - anche quando agli occhi ingenui del profano può sembrare statica e immutabile - una forma in movimento nel tempo (e nello spazio) da cui può sorgere inatteso e imprevedibile un senso interpretativo inedito. Ma se la forma è un organismo vivente, e in quanto tale sempre tra-

7. Ibid. Per le citazioni seguenti ibid.

8. Come sottolinea Carlo Ettore COLOMBO (Les débuts italo-provençaux..., op. cit., p. 34-35), un vivo interesse per le lingue caratterizza gli studi di Contini negli anni 1930 : prima a Torino con Santorre Debenedetti, poi a Parigi con Clovis Brunel, viene introdotto alla linguistica diacronica e alla dialettologia. In quegli anni le questioni del bilinguismo e della diglossia - sollevate alla fine dell'Ottocento dagli ellenisti francesi Jean Psichari e Hubert Pernot - sono di nuovo oggetto a Parigi di accesi dibattiti (v. Lambert-Félix PRUDENT, Diglossie et interlecte, in Jean-Baptiste MARCELLESI (dir.), "Bilinguisme et diglossie », Langages, a. 15, 6 (1981), p. 15, 18). Ecco perché il Contini filologo userà il plurilinguismo dei testi letterari come criterio linguistico pertinente per categorizzare e interpretare le poetiche, antiche o moderne, in gioco.

9. Concezione della traduzione che ritroviamo all'inizio degli anni 1920 sia in Walter Benjamin che in Giovanni Gentile, v. Viviana AGOSTINI-OUAFI, Poetiche della traduzione, cit., p. 35-38; per un più approfondito sviluppo della questione, v. anche $I D$., " La traduction et le fascisme : quelques réflexions à partir des théories de Croce et Gentile » [2010], in Iulia COSMA (dir.), Translationes, 7 (2015) : Stages of the History of Translation, in part. p. 40-44. Sull'omologia del tradurre con l'interpretare v. anche Hans-Georg GADAMER, «Dall'ermeneutica all'ontologia ", in Siri NERGAARD (dir.), Teorie contemporanee della traduzione, Milano, Bompiani, 1995, p. 347. Molto critico invece rispetto a questa identità è Umberto Eco che, a scanso di equivoci, provocatoriamente intitola Interpretare non è tradurre il capitolo di Dire quasi la stessa cosa da lui dedicato all'approccio ermeneutico della traduzione (Milano, Bompiani, 2003, p. 225-253).

10. Gianfranco CONTINI, Esercizio d'interpretazione sopra un sonetto di Dante [1947], in Varianti e altra linguistica, op. cit., p. 161-168.

11. Jorge Louis BORGES, Pierre Menard, autore del Chichiotte [1939], in Finzioni, trad. it. F. Lucentini, Torino, Einaudi, $1978^{4}$, p. 43-44. 
ducibile, ciò significa che il piano dell'espressione e quello dell'esegesi sono così strettamente collegati da abolire nella loro simbiosi il dualismo aristotelico di forma e contenuto ${ }^{12}$.

Sono in verità le occorrenze sistemiche e contestualizzate della stessa forma linguistica nel macrotesto autoriale che permettono, secondo Contini, di offrire per ogni reiterazione di un lemma o di un sintagma una "traduzione meno imprecisa $"^{13}$. Dopo aver dimostrato, citando varie occorrenze dantesche, che l'arcaismo ormai del tutto scomparso labbia si differenzia dal tuttora esistente faccia e significa piuttosto, nel sonetto suddetto, fisionomia, Contini afferma : "Tradurre non significa infatti altro se non determinare il nuovo rapporto dei sinonimi e affini nella cultura rappresentata dalla nostra lingua, la nuova ripartizione, per dir così, in parole della realtà che si considera come oggettiva e costante $»^{14}$. È evidente che per Contini la realtà non è né oggettiva né costante ma, come la vita, un inarrestabile e ineffabile fluire. Quanto al suo approccio dello sviluppo espressivo, al contempo filologico (Growth) e sistemico (Structure), condotto come un'operazione ermeneutico-traduttiva, esso ci interessa in sommo grado perché il filologo-traduttore Pézard, traducendo Dante in francese, ha dovuto affrontare un'operazione non solo endolinguistica ma anche e soprattutto interlinguistica, passando dall'italiano antico e dal latino medievale delle varie opere di Dante al francese moderno dei suoi lettori. Le scelte da lui fatte, soprattutto la decisione di costellare il testo di arcaismi, hanno suscitato non poche reazioni e polemiche ${ }^{15}$.

Lavorando in varie fasi intermittenti, dal 2008 al 2012, nel fondo Pézard del Collège de France depositato all'IMEC ${ }^{16}$, abbiamo constatato l'esistenza di alcune lettere di Contini che risalgono agli anni 1960, di cui due relative in particolare a pubblicazioni di Pézard nella rivista fiorentina "Studi danteschi » diretta all'epoca dallo stesso Contini ${ }^{17}$. Siccome eravamo totalmente presi dallo studio degli innumerevoli e straordinari scartafacci traduttivi danteschi disponibili in questo fondo, non abbiamo pensato subito di consultare tale corrispondenza. Quando abbiamo deciso di farlo, il fondo del Collège de France era ormai partito a Pierrefitte-sur-Seine (Archives Nationales) e a detta dell'archivista, novellamente incaricato di rifare l'inventario di quello e di altri fondi pezardiani colà riuniti, tali archivi non saranno più consultabili per vari mesi : si potrà farlo, forse, nel 2017? Per nostra fortuna avevamo preso all'IMEC un po' di appunti, relativi al catalogo pezardiano del Collège de France, e rileggendoli ci è venuto il dubbio che la relazione Contini-Pézard fosse uno dei capitoli più importanti, ancora quasi tutto da scrivere, delle relazioni linguistico-letterarie franco-italiane del periodo 1960-1980.

Esistono in effetti in tale fondo tre presentazioni di Gianfranco Contini, la prima del 1975 e l'ultima del 1980, scritte da Pézard allo scopo di farlo eleggere come membro associato straniero (Associé Étranger, AE) all'Institut de France ${ }^{18}$. Grazie al fruttuoso terzo tentativo di Pézard, Contini è stato effettivamente eletto, a maggioranza assoluta dei suffragi espressi, come AE dell'Académie des Inscriptions et Belles-Lettres il 30 maggio 1980, in sostituzione del deceduto Nastase Orlandos ${ }^{19}$.

12. Per una critica della dualità del segno linguistico dal punto di vista traduttologico, v. Henri MESCHONNIC, Poétique du traduire, Lagrasse, Verdier, 1999, p. 115, 117, 142-143, 190 et passim.

13. Gianfranco CONTINI, Esercizio d'interpretazione..., cit., p. 162.

14. Ibid.

15. V. Viviana AgOstini-OuAfI, "André Pézard traducteur de Dante ou le choix inactuel de l'archaïsme ", Transalpina, 18 (2015), cit., p. 125-140.

16. Institut Mémoires de l'Édition Contemporaine, con sede nell'abbaye d'Ardenne, presso Caen.

17. André PÉZARD, "Ce qui gronde en l'éternel " (Inf. VI 94-115), Studi danteschi, 42 (1965), p. 207-233 e ID., "Deux notes sur Uguccione de Pise et la faute de Priscien ", Studi danteschi, 45 (1968), p. 157-165.

18. Nel vecchio catalogo dell'IMEC : Pzd. 14.15. "Trois présentations du Prof. G. Contini (1975-1980)".

19. V. Ordre du jour, in Comptes rendus des séances de l'Académie des Inscriptions et Belles-Lettres, a. 124, 2 (1980), p. 393, http://www.persee.fr/doc/crai_0065-0536_1980_num_124_2_18775; ed anche Gilbert LAZARD, Allocution à l'occasion du décès de M. Gianfranco Contini, associé étranger de l'Académie, Ibid., a. 134, 1 (1990), p. 197-198, http://www.persee.fr/doc/crai_0065-0536_1990_num_134_1_14833. 
Cosa mai avrà scritto Pézard, in questa terza presentazione, per convincere l'Académie, riunitasi a tal scopo in " comitato segreto $"^{20}$ ? Nel fondo archivistico ora per noi inaccessibile avevamo anche trovato traccia di una recensione pezardiana ai Poeti del Duecento uscita sulla prestigiosa rivista parigina " Romania », dal 1872 specializzata nello studio delle lingue e letterature romanze ${ }^{21}$ : la data di pubblicazione è particolarmente interessante, 1962, giacché all'epoca Pézard stava alacremente lavorando alla sua traduzione dell'opera completa, latina e italiana, di Dante ${ }^{22}$. Il tono generale con cui evoca nella recensione il lavoro filologico di Contini è quello ammirativo e rispettoso che ritroviamo poi anche nel suo terzo Avertissement della Pléiade (quello del 31 dicembre 1975) quando afferma di attendere con impazienza l'edizione definitiva e annotata del Fiore, intrapresa da Contini sulla base di una sua " tesi geniale", per poter a sua volta tradurlo e includerlo nel volume della Pléiade ${ }^{23}$. La riflessività del filologo francese, titolare della cattedra di letteratura e civiltà italiana al Collège de France dal 1951 al 1963, è sempre in tutti i suoi scritti di alto livello ed espressa con sincerità. Una buona recensione è fatta prima di tutto per trasmettere informazioni significative e pertinenti sull'opera recensita, ma può altresì svelare la postura e i criteri ermeneutici, linguistici e letterari del recensore. La presentazione e il commento pezardiani ai Poeti del Duecento hanno da questo punto di vista ben soddisfatto entrambi le nostre attese.

Cosa pensava Pézard di questo collega transalpino, dei suoi studi e del suo metodo di lavoro? In sintesi, egli definisce i Poeti del Duecento " un meraviglioso strumento di lavoro ${ }^{24}$ in cui "si cerca invano ciò che si potrebbe togliere, o cambiare ». Per lui il volume continiano costituisce " un festino così notevole " per qualità ed abbondanza che la " fame » di colui che desidera altre portate dopo un così lauto banchetto può sembrare «indecente ». E subito specifica che è proprio il suo caso, e che la sua è una "fame antica ", di lunga data, nata quando Contini, "nel 1946 ", pubblicò la sua " eccellente raccolta di tutte le Rime "sparse" di Dante ${ }^{25}$. Così, tra le righe del suo commento, Pézard ci dice persino quando la scoperta dell'approccio filologico di Contini ha destato il suo più vivo interesse, o, per continuare nella metafora barocca da lui usata, la sua voglia irrefrenabile di nutrirsi del sapere dantesco di Contini. Possiamo quindi stabilire un terminus ad quo nella relazione PézardContini che è il 1946 : Pézard ha appena discusso la sua tesi di dottorato sul canto XV dell'Inferno ed ottenuto la cattedra alla Faculté de Lettres di Lione. Ha già in Francia nel 1931 curato un'edizione in italiano de La Vita Nova, e nel 1940 pubblicato un volume in francese sul Convivio di Dante. Ora, dopo aver evocato il suo primo entusiasmante incontro con Contini, egli sottolinea subito che le Rime e i Poeti del Duecento sottostanno, a distanza di quattordici anni, agli stessi principi organizzativi e metodologici, ovvero sono concepiti con "lo stesso spirito, lo stesso metodo ${ }^{26}$ e ne enumera le caratteristiche : cappello introduttivo prima di ogni testo o gruppo di testi, « note filologiche e letterarie brevi, dense, penetranti ", nota finale al testo, tradizione manoscritta e bibliografia essenziale.

20. Le citazioni in italiano di testi pubblicati in francese saranno, come in questo caso, sempre da noi tradotte.

21. André PÉZARD, "Compte rendu : Poeti del Duecento, a cura di Gianfranco Contini " (La letteratura italiana, storia e testi, vol. 2; Milano-Napoli, Ricciardo Ricciardi ed. 1960; deux tomes in $-8^{\circ}$, XXVI + 932 et 1002 pages), Romania, a. 91, 3 (1962), p. 412-419.

22. DANTE, Euvres complètes. Traduction et commentaires par André Pézard [1965], Paris, Gallimard, "Bibliothèque de la Pléiade ", $1983^{5}$.

23. André PÉZARD, Avertissement de la troisième édition, in DANTE, Euvres complètes, cit., p. XLVII-XLVIII. Abbiamo tradotto con un ricco apparato di note, e pubblicato in Italia, non solo la prima avvertenza del 1965, ma anche quelle aggiunte alle edizioni successive dell'opera (1967, 1975, 1979, compresa l'ultima del 1983), v. André PÉZARD, Dante e il pittore persiano. Note sul tradurre, introd., trad., ed. Viviana Agostini-Ouafi, postfazione Jean-Yves Masson, Modena, Mucchi, 2014 (per il riferimento a Contini, ibid., p. 102).

24. Recensione di André Pézard a Poeti del Duecento..., cit., p. 415. Per le citazioni seguenti Ibid.

25. Si tratta della seconda edizione delle Rime curata da Contini (apparsa nella collana Nuova raccolta di classici italiani annotati); la prima edizione, pubblicata sempre a Torino da Einaudi, era quella del 1939.

26. Ibid., p. 416. 
Mancano però ancora secondo lui nei due voluminosi tomi dei Poeti del Duecento, sebbene in minor misura rispetto alle Rime del 1946, vari componimenti che senza alcun dubbio sono in relazione con l'opera di Dante. Ma la delusione provata nel non trovare in quest'edizione tutto ciò che avrebbe desiderato leggervi, non è di fatto una critica. Un tale lavoro è ai suoi occhi esemplare : costituisce uno sprone stimolante e sapiente per penetrare, come non si era mai fatto prima, nell'opera di Dante.

Per comprendere l'estrema attenzione filologica portata da Pézard all'apparato formale ed ermeneutico del doppio volume continiano, ma anche per dare il giusto peso al rimpianto da lui esplicitamente espresso di non vedervi inclusi alcuni autori o alcuni testi - malgrado secondo lui la mole enorme di materiale in esso riproposto in veste aggiornata o presentato per la prima volta in modo qualitativamente ineccepibile -, occorre tener conto della tempistica in cui tale recensione si inserisce. In effetti, varie note sui ritmi e le fasi del lavoro traduttorio che sta realizzando, scritte da Pézard in margine ai suoi manoscritti autografi, ci permettono di ipotizzare che, quando recensisce questo lavoro di Contini, ha già trascritto a macchina la sua traduzione della Vita Nova e delle Rime ${ }^{27}$. Il valore scientifico dei testi pubblicati risiede per Pézard innanzi tutto nel fatto che Contini non si è accontentato delle edizioni critiche esistenti, anche recenti, ma ha rivisto tutti quei testi sui loro manoscritti migliorandone la grafia o la punteggiatura, correggendo il taglio o l'integrità di certi gruppi verbali, restaurando con sicurezza i prestiti fino ad allora non identificati provenienti dai dialetti, dal francese e dal provenzale antichi (restauro in cui Contini ai suoi occhi eccelle). Secondo lui si tratta di un lavoro critico " ardito ${ }^{28}$ giacché, facendo leva su " mezzi minuti e poco rumorosi " come i manoscritti, Contini " tende a rompere pigre tradizioni interpretative ». Ma " questo maestro dotto, sensibile e cortese " non prende decisioni sommarie e non dà per certo ciò che è solo "allettante o verosimile ». Per Pézard la modestia, la discrezione e la prudenza ermeneutica del filologo italiano sono frutto del suo metodo di lavoro, che implica in sé un'esigente deontologia, ma - e ciò vale ugualmente per Pézard - modestia, discrezione e prudenza sono anche il risultato secondo noi di un vasto sapere, poiché solo chi sa, sa socraticamente di non sapere.

Sul metodo di Contini poi Pézard afferma :

Se succede a Contini - e gli succede spesso - di rinnovare il modo d'intendere un verso, e anche una parola sempre viva ma per il lungo uso sviata dal suo valore antico, oppure una parola caduta in disuso, alterata nella sua forma e perciò rivestita arbitrariamente d'un senso che non le si addice, la critica testuale più scrupolosa precede sempre la critica interpretativa. ${ }^{29}$

Solo quest'ultima affermazione meriterebbe un esteso commento ma ci limiteremo a sottolineare come essa sia in sintonia con l'avvicinamento, già citato, della linea espressiva e della linea esegetica che Contini traccia nel suo scritto del 1965 Filologia ed esegesi dantesca.

Pézard osserva poi che la critica filologica continiana non sommerge e mortifica il suo lettore con un apparato critico sovrabbondante e « troppo spesso sterile». La funzione discreta di tale apparato è di motivare la correzione proposta, tanto più se essa rompe con la tradizione interpretativa vigente : "brevi note, a piè di pagina, giustificano le novità a prima vista più sconcertanti ", ma secondo Pézard si tratta di una " erudizione già assorbita e digerita " da Contini, quindi in grado di aiutare il lettore in modo rapido, costante e sicuro. Questo tipo di apparato pedagogico caratterizzerà anche l'opera completa di Dante in francese : Pézard terrà anzi particolarmente alla presenza delle note a

27. V. nella seconda stesura manoscritta della canzone Donna Pietosa: Pzd 10.12. Vie Nouvelle, XX, f. 3v., scritto con una biro blu, sul margine sinistro, " 14 avril 1960 » e subito sotto, con una biro rossa, "recopié à la machine le 11 mars 1961 ".

28. Recensione di André PÉZARD a Poeti del Duecento..., cit., p. 413.

29. Ibid. La sottolineatura è nostra. 
piè di pagina (invece che a fine volume, come sarebbe tradizione nella Pléiade ${ }^{30}$ ). Per questo aspetto il modello critico testuale ed esegetico continiano sarà per lui un punto di riferimento essenziale. Anche certi segni diacritici introdotti da Contini, in particolare il punto in alto usato dai provenzalisti e che indica per la prima volta, nei Poeti del Duecento, il raddoppiamento fonosintattico tipico del toscano antico e moderno, sono considerati da Pézard non un sovrappiù erudito o inutile d'interpunzione, ma "glosse sommarie " ${ }^{31}$ che permettono di cogliere con una sola occhiata la spiegazione proposta da Contini di un preciso passaggio testuale. Ritroveremo questi spunti metodologici in certi spazi vuoti sintagmatici della sua traduzione (stratagemma ritmico-semantico usato poi anche dal Meschonnic traduttore biblico dove però le ampie spaziature tra gli emistichi rimpiazzano la punteggiatura) e nell'uso in nota della doppia parentesi per richiamare l'attenzione del lettore su interpretazioni nuove e azzardate da lui proposte del testo dantesco ${ }^{32}$.

Pézard loda poi esplicitamente, visto il modo in cui Contini ha trattato le questioni ortografiche, " lo scrupolo di discrezione " $^{33}$ già osservato per la punteggiatura. Invece di operare come certi editori una uniformizzazione fonologica dell'idioletto degli autori, col pretesto di rispettare un tratto dialettale, arcaico o classico, Contini non fa scomparire gli elementi bizzarri o incerti che permettono di « congetturare un'alterazione ancora insospettata, d'intravedere una variante di senso ». Sensibile alla libertà espressiva di ogni scrittore, al temperamento e al pensiero di ciascun poeta, Contini rifiuta "l'allineamento militare delle forme » a delle "norme generali». Pur sapendo che le sue scelte lasciano scontenti « i dottrinari della filologia », Contini « lascia al lettore, informato di tutti i possibili, il tempo e la libertà ("le loisir») di valutare e scegliere il probabile ». Per le irregolarità metriche, Contini applica lo stesso atteggiamento critico, e Pézard se ne rallegra : in effetti, pur se molte cose ancora non sono chiare nell'esegesi dantesca, secondo quest'ultimo "bisogna saper ignorare ".

Si evince chiaramente da queste affermazioni di Pézard che egli si schiera con Contini contro quella corrente della filologia, periferica ormai ma ancora attiva negli anni 1960, rigidamente codificata e arroccata nella sua ortodossia. Egli lo fa in nome di una problematizzazione dell'esegesi filologica rispettosa non solo dei testi manoscritti ma anche dell'intelligenza del proprio lettore. La critica testuale non deve né semplificare né irreggimentare i testi tràditi per guidare a senso unico un lettore considerato di fatto pigro e immaturo. Nel suo Avertissement della Pléiade ritroveremo la centralità di quest'ultimo aspetto, da noi sintetizzato (alludendo a Umberto Eco) con l'espressione "Lector in fabula " ${ }^{34}$. Ora, quest'idea di lettore attivo che presuppone il metodo di Contini (ma anche quello di Pézard) concerne pure il filologo-interprete in quanto prima di tutto lettore. Perciò Pézard soggiunge che è inutile cercare, nell'estrema finezza dell'" arte critica ${ }^{35}$ di Contini, le formule comode che soddisfino $\mathrm{i}$ " pedanti " e dispensino ormai " il gregge » dei seguaci dall'atto del riflettere. Come attesta il termine espressamente dispregiativo " gregge " ( les ouailles»), la polemica contro la corrente filologica ortodossa raggiunge qui un tono così vivo che quasi ci sorprende. Pézard sottolinea subito che "le grandi teorie, i quadri sistematici e il vocabolario speciale " sono concetti, schemi, termini che si possono presto afferrare a un maestro, o modificare a un rivale, ma che la forza e l'originalità dell'arte critica di Contini si trovano altrove : "Egli si preoccupa meno di definire e di opporre, per date e frontiere, dei periodi, degli ambienti - più o meno convenzionali - che di attivare, alla luce della sua esperienza letteraria, degli aspetti umani».

30. André PÉZARD, Dante e il pittore persiano. Note sul tradurre, cit., p. 87.

31. Recensione di André PÉZARD a Poeti del Duecento..., cit., p. 414.

32. Sugli spazi bianchi e le doppie parentesi tonde, v. André PÉZARD, Dante e il pittore persiano. Note sul tradurre, cit., p. $54-55$ e p. 86.

33. Recensione di André PÉZARD a Poeti del Duecento..., cit., p. 414.

34. André PÉZARD, Dante e il pittore persiano. Note sul tradurre, cit., p. 29-30. V. la nostra introduzione, p. 24.

35. Recensione di André PÉZARD a Poeti del Duecento..., cit., p. 415. 
In effetti un altro punto fondamentale che si evince da tali affermazioni è la dimensione poetico-espressiva dell'esegesi filologica : agli occhi di Pézard, Contini restaura e interpreta i testi non solo con la sapienza e la precisione del critico testuale, ma anche con la sensibilità artistica profonda del poeta e critico letterario. La dimensione strutturalista e poetica della filologia continiana permea totalmente, fin dall'incipit dell'Avertissement della Pléiade, la riflessione traduttologica di Pézard. A più riprese Contini afferma in Filologia ed esegesi dantesca che l'obbiettivo più importante del sistema delle concordanze è quello di permettere di «spiegar Dante con Dante ${ }^{36}$. Pézard negli stessi anni giustifica la sua decisione di aver voluto tradurre da solo l'opera completa di Dante perché convinto che solo il traduttore unico, purché dotato di buona memoria, può collegare, per esempio, " ciò che è detto nel trattato politico e ciò che si intuisce nel sonetto d'amore, tanto distanti l'uno dall'altro per gli anni, per la forma e l'essenza, e che tuttavia fanno uso di termini analoghi ${ }^{37}$. La posizione privilegiata del traduttore unico permette a costui di « cercare i rapporti segreti che in una stessa testa, la testa meravigliosa di Dante, esistono necessariamente tra due pagine illuminate da uno stesso fuoco ". Lo strutturalismo filologico ed ermeneutico pézardiano si pone costantemente come fine il rispetto del pensiero dottrinale, della sensibilità poetica e dello stile musicale di Dante : numerosi sono i passaggi dell'Avertissement in cui egli giustifica il suo operato evocando ciò che il poeta, nell'una o nell'altra delle sue opere, ha affermato sull'argomento trattato, in primis la musicalità poetica del tradurre, la simbiosi inscindibile forma-senso ${ }^{38}$. Mai il traduttore pretende di sostituire la sua concezione dell'opera poetica a quella dell'autore, anzi Pézard, come Contini, afferma perentoriamente : "Mi sono rivolto, per comprendere Dante e trapiantarlo in Francia, a Dante stesso e ai grandi italiani che lo hanno illustrato con le loro glosse $"^{39}$.

Ora sappiamo, grazie anche alla recensione di Pézard da noi analizzata, quale fosse ai suoi occhi il posto occupato da Contini tra questi grandi italiani. Ma il loro sodalizio, rafforzatosi come abbiamo dimostrato negli anni 1960-1970, ci dice anche che il loro dotto approccio critico, storicizzante e legato alla lettura filologica dei testi, non era ingabbiabile in nessuna teoria sincronica, rigida e schematica : per nostra fortuna, questi due grandi critici erano già post-strutturalisti.

36. Gianfranco CONTINI, Varianti e altra linguistica, cit., p. 427. V. anche ibid., p. 428 : «la spiegazione di Dante con Dante".

37. André PÉZARD, Dante e il pittore persiano. Note sul tradurre, cit., p. 28.

38. Ibid., p. 46-47. V. la nostra introduzione, ibid., p. 15-17.

39. Ibid., p. 88. 